\title{
An exotic class of Kaluza-Klein models
}

\author{
Matt Visser \\ Physics Department, \\ University of Southern California, \\ Los Angeles, CA 90080-0484, \\ $U S A$
}

12 September 1985; LATEX-ed February 1, 2008

\begin{abstract}
We discuss an exotic class of Kaluza-Klein models in which the internal space is neither compact nor even of finite volume. Rather than using the usual compact internal space we consider the case where particles are gravitationally trapped near a four-dimensional submanifold of the higher dimensional spacetime. A specific model exhibiting this phenomenon is constructed in five dimensions.

Physics Letters B159, 22-25 (1985).

Note: This rather old paper has recently been subject of renewed interest due to the explosion of activity in the "non-compact extra dimensions" variant of the Kaluza-Klein model. It is not available elsewhere on the Internet, and in the interests of easy access I am placing it on hep-th. The body of the paper is identical to the published version. A small separate note has been added at the end of the paper.

Present address:

Physics Dept, Washington University, St Louis, MO 63130-4899

E-mail: visser@kiwi.wustl.edu

Homepage: http://www.physics.wustl.edu/visser

Archive: hep-th/9910093
\end{abstract}


Introduction. In Kaluza-Klein theories of the ordinary type spacetime is assumed to be of the form $M_{4} \times K ; M_{4}$ a four-dimensional manifold and $K$ a compact manifold of "internal" co-ordinates. Classically particles are free to move around on $K$, thus $K$ must be assumed to be very small. The alternative we wish to begin to explore in this note is to somehow arrange for an absence of translational invariance in the extra "internal" directions, and then use gravity to trap particles near a four-dimensional submanifold. Suggestions along similar lines (without gravity) have been made by Rubakov and Shaposhnikov [1]. For simplicity we consider the case of five dimensions, though the general procedure immediately generalizes to arbitrary dimensionality. We first give a general discussion that does not specify the particular form of the matter giving rise to the assumed metric, and only later do we exhibit a particular example. The particular example we discuss also exhibits a nice mechanism for generating a zero effective cosmological constant from a nonzero five-dimensional cosmological constant.

The Metric. Let the co-ordinates be $X^{A}=(t, x, y, z, \xi) ; A=0,1,2,3,5$. Assume that the metric is of the form

$$
g=-e^{2 \phi(\xi)}(\mathrm{d} t)^{2}+\mathrm{d} \vec{x} \cdot \mathrm{d} \vec{x}+(\mathrm{d} \xi)^{2} .
$$

Without even having to look at field equations it is immediately apparent that there are four Killing vectors and, therefore, four constants of the motion for particles following geodesics

$$
\begin{gathered}
E=-\left(P, \frac{\partial}{\partial t}\right)=-P^{A} g_{A B}\left(\frac{\partial}{\partial t}\right)^{B}=P^{0} e^{2 \phi} \\
p^{i}=\left(P, \frac{\partial}{\partial x^{i}}\right)=P^{A} g_{A B}\left(\frac{\partial}{\partial x^{i}}\right)^{B}=P^{i} \quad[i=1,2,3] .
\end{gathered}
$$

A particle having a definite rest mass in the five dimensional sense now satisfies

$$
-\left(M_{5}\right)^{2}=P^{A} g_{A B} P^{B}=-e^{2 \phi}\left(P^{0}\right)^{2}+\vec{p} \cdot \vec{p}+\left(P^{5}\right)^{2} .
$$

Consequently

$$
P^{5}=\sqrt{E^{2} e^{-2 \phi}-\left(M_{5}\right)^{2}-\vec{p}^{2}}
$$

with $(E, \vec{p})$ constants of the motion. Thus if

$$
E<\sqrt{\left(M_{5}\right)^{2}+\vec{p}^{2}} \sup \left(e^{\phi}\right)
$$


any classical particle will be bound by the potential $\phi(\xi)$. The experimental nonobservation of any extra dimensions implies that $e^{\phi}$ must rise very rapidly, presumably with a length scale of order the Planck mass.

To see how this gravitational trapping works quantum mechanically consider the Klein-Gordon equation in such a background metric:

$$
\frac{1}{\sqrt{-g}} \partial_{A}\left(\sqrt{-g} g^{A B} \partial_{B} \Psi\right)=\left(M_{5}\right)^{2} \Psi .
$$

Substituting the explicit form of the metric

$$
\left[-e^{-2 \phi}\left(\frac{\partial}{\partial t}\right)^{2}+\left(\frac{\partial}{\partial \vec{x}}\right)^{2}+\left(\frac{\partial}{\partial \xi}\right)^{2}\right] \Psi+\left(\frac{\partial \phi}{\partial \xi}\right) \frac{\partial \Psi}{\partial \xi}=\left(M_{5}\right)^{2} \Psi .
$$

Try a separation of variables

$$
\Psi=\exp [-i(\omega t-\vec{k} \cdot \vec{x})] e^{-\phi / 2} \Psi_{\perp}(\xi) .
$$

Then $\Psi_{\perp}(\xi)$ satisfies the Schrodinger equation

$$
\left[-\frac{1}{2}\left(\frac{\partial}{\partial \xi}\right)^{2}+\frac{1}{2}\left(\frac{1}{2} \phi^{\prime \prime}+\frac{1}{4} \phi^{\prime} \phi^{\prime}-\omega^{2} e^{-2 \phi}\right)\right] \Psi_{\perp}=-\frac{1}{2}\left[\left(M_{5}\right)^{2}+\vec{k}^{2}\right] \Psi_{\perp} .
$$

Rewrite the eigenvalue problem as

$$
\left[-\frac{1}{2}\left(\frac{\partial}{\partial \xi}\right)^{2}+V(\omega, \xi)\right] \eta_{n}(\omega, \xi)=-\lambda_{n}(\omega) \eta_{n}(\omega, \xi)
$$

with

$$
V(\omega, \xi)=\frac{1}{4} \phi^{\prime \prime}+\frac{1}{8} \phi^{\prime} \phi^{\prime}-\frac{1}{2} \omega^{2} e^{-2 \phi} .
$$

Then the solutions of the Klein-Gordon equation are travelling waves in the four usual directions but bound states in the fifth co-ordinate

$$
\Psi(t, \vec{x}, \xi)=\exp [i(\omega t-\vec{k} \cdot \vec{x})] e^{-\phi / 2} \eta_{n}(\omega, \xi) .
$$

The excitation spectrum is found by solving

$$
2 \lambda_{n}(\omega)=\left(M_{5}\right)^{2}+(\vec{k})^{2} .
$$


Note that by choosing an appropriate normalization for $t$ we can get

$$
\inf (\phi)=0 .
$$

Then $V(\omega, \xi)$ is bounded by

$$
V(\omega=0, \xi) \geq V(\omega, \xi) \geq V(\omega=0, \xi)-\frac{1}{2} \omega^{2} .
$$

Leading to the inequality

$$
\lambda_{n}(\omega) \leq \lambda_{0}(\omega=0)+\frac{1}{2} \omega^{2} .
$$

In the usual type of Kaluza-Klein model one obtains an infinite tower of excited states of ever increasing rest mass. For this exotic class of KaluzaKlein models the spectrum is significantly more complex. Let $\lambda_{n}^{-1}(x)$ be the inverse function to $x=\lambda_{n}(\omega)$, then

$$
\omega_{n}(\vec{k})=\lambda_{n}^{-1}\left(\frac{1}{2}\left[\left(M_{5}\right)^{2}+\vec{k}^{2}\right]\right) .
$$

In particular the spectrum will not in general be Lorentz invariant (i.e., will not have a $O(1,3)$ symmetry). This should not be surprising since our ansatz for the metric did not possess an $O(1,3)$ invariance. We would however like to see $O(1,3)$ invariance show up in some approximation. To achieve this let us define $\Omega_{n}$ by

$$
\left.2 \lambda_{n}\left(\Omega_{n}\right)=\left(M_{5}\right)^{2} ; \quad \text { (i.e., } \vec{k}=0\right) .
$$

Now evaluate $\lambda_{n}(\omega)$ near $\omega=\Omega_{n}$ by using first-order perturbation theory in $\omega^{2}$; we see

$$
\begin{aligned}
\lambda_{n}(\omega) & =\lambda_{n}\left(\Omega_{n}\right)+\frac{1}{2} c_{n}^{-2}\left(\omega^{2}-\Omega_{n}^{2}\right)+\ldots, \\
c_{n}^{-2} & =\left\langle\eta_{n}\left(\Omega_{n}, \xi\right)\left|e^{-2 \phi(\xi)}\right| \eta_{n}\left(\Omega_{n}, \xi\right)\right\rangle .
\end{aligned}
$$

The spectrum may now be approximated by solving

$$
\left(M_{5}\right)^{2}+c_{n}^{-2}\left(\omega^{2}-\Omega_{n}^{2}\right)+\ldots=\left(M_{5}\right)^{2}+\vec{k}^{2} .
$$

Then

$$
\omega^{2}=\Omega_{n}^{2}+c_{n}^{2} \vec{k}^{2}+\ldots
$$


Thus in the region where first order perturbation theory is valid [this region depends on the precise form of $\phi(\xi)$ ] we obtain an approximately Lorentz invariant spectrum with rest energy $\Omega_{n}$ and "effective speed of light" $c_{n}$. (In General Relativity it is rather common to interpret $c=\sqrt{g_{00}}$ as the "local speed of light", observe that $c_{n}^{-2}=\left\langle c(\xi)^{-2}\right\rangle$ is just the expectation value of this local speed of light for the $n$ 'th excited mode).

Up to this point we have nowhere used the Einstein field equations, the discussion has accordingly been very general and qualitative.

The Field equations. Starting from the metric:

$$
\begin{gathered}
g_{00}=-e^{2 \phi(\xi)} ; \\
g_{11}=g_{22}=g_{33}=g_{55}=1 ;
\end{gathered}
$$

(all other components zero); it is a simple matter to compute the Ricci tensor and Einstein tensor

$$
R_{0}^{0}=R_{5}^{5}=-\phi^{\prime \prime}-\phi^{\prime} \phi^{\prime}=-e^{-\phi}\left(e^{\phi}\right)^{\prime \prime},
$$

all other components vanish;

$$
G^{11}=G^{22}=G^{33}=+e^{-\phi}\left(e^{\phi}\right)^{\prime \prime},
$$

all other components vanish.

Consider the Einstein field equations in the form

$$
G_{A B}=+\lambda g_{A B}+T_{A B},
$$

we have

$$
\begin{aligned}
& \text { 1) } T_{0}^{0}=-\lambda=T_{5}^{5}, \\
& \text { 2) }\left(e^{\phi}\right)^{\prime \prime}=\left(+\lambda+T_{11}\right) e^{\phi}, \\
& \text { 3) } T_{11}=T_{22}=T_{33}
\end{aligned}
$$

other components vanish.

So that we see that it is the pressure $p=T_{11}=T_{22}=T_{33}$ that is responsible for driving the $\xi$ dependence of $\phi$, while the density must be independent of $\xi$.

In fact, a stress energy tensor of this type can easily be constructed by considering five-dimensional Electromagnetism with a constant electric field pointing in the $\xi$ direction. Take:

$$
\mathcal{L}=-\frac{1}{4} F_{A B} F^{A B} .
$$


Exotic Kaluza-Klein...

$$
T_{A B}=-2 \frac{\delta \mathcal{L}}{\delta g^{A B}}+g_{A B} \mathcal{L}=F_{A C} F_{B}^{C}-\frac{1}{4} g_{A B}\left(F_{C D} F^{C D}\right) .
$$

Pick

$$
A_{0}=a(\xi), \quad A_{1}=A_{2}=A_{3}=A_{5}=0 .
$$

Then

$$
F_{05}=-F_{50}=a^{\prime}, \quad F_{A B} F^{A B}=-2 e^{-2 \phi}\left(a^{\prime}\right)^{2} .
$$

For convenience define

$$
E=e^{-\phi} a^{\prime}
$$

Now

$$
\begin{aligned}
T_{0}^{0} & =F_{05} F^{05}-\frac{1}{4}\left(-2 E^{2}\right) \\
& =a^{\prime}\left(-e^{-2 \phi} a^{\prime}\right)+\frac{1}{2} E^{2} \\
& =-\frac{1}{2} E^{2} .
\end{aligned}
$$

Similarly

$$
T_{5}^{5}=-\frac{1}{2} E^{2} ; \quad T_{1}^{1}=T_{2}^{2}=T_{3}^{3}=+\frac{1}{2} E^{2} .
$$

The field equations now yield

$$
\lambda=+\frac{1}{2} E^{2}, \quad\left(e^{\phi}\right)^{\prime \prime}=E^{2}\left(e^{\phi}\right),
$$

with solution

$$
e^{\phi}=\cosh (E \xi), \quad a=\sinh (E \xi) .
$$

Note that the equation of motion for $F$ is

$$
\begin{aligned}
\frac{1}{\sqrt{-g}} \partial_{A}\left(\sqrt{-g} F_{A B}\right)=0 & \rightarrow \partial_{5}\left(e^{\phi}\left[-e^{-2 \phi} a^{\prime}\right]\right)=0 \\
& \rightarrow \partial_{5}\left(e^{-\phi} a^{\prime}\right)=0 \\
& \rightarrow \partial_{5}(E)=0
\end{aligned}
$$

consistent with the result $\lambda=+\frac{1}{2} E^{2}$.

\footnotetext{
${ }^{1}$ Note added: Here and henceforth $E$ is the electric field in the 5 direction, not the particle energy.
} 
What has happened in this model is that a five-dimensional cosmological constant has conspired with a constant electric field $E=\sqrt{2 \lambda}$, to yield a four-dimensional submanifold of zero four-dimensional cosmological constant, together with a potential $\phi=\ln \cosh (E \xi)$ that traps particles in the fifth direction. The quantum mechanical trapping is in this case governed by the Schrodinger equation

$$
\left[-\frac{1}{2}\left(\frac{\partial}{\partial \xi}\right)^{2}+\frac{1}{8} E^{2}-\left(\frac{1}{2} \omega^{2}-\frac{1}{8} E^{2}\right) \operatorname{sech}^{2}(E \phi)\right] \eta_{n}=\lambda_{n} \eta_{n} .
$$

This potential is the well-known Rosen-Morse potential, the exact eigenvalues are known to be [2]

$$
\lambda_{n}=\frac{1}{2}\left\{\left(\omega-\left[n+\frac{1}{2}\right] E\right)^{2}-\left(\frac{E}{2}\right)^{2}\right\} .
$$

We solve for the exact spectrum

$$
\omega_{n}(\vec{k})=\left[n+\frac{1}{2}\right] E \pm\left[\left(\frac{E}{2}\right)^{2}+\left(M_{5}\right)^{2}+(\vec{k})^{2}\right]^{1 / 2} .
$$

We define a 4-dimensional mass by

$$
\left(m_{4}\right)^{2}=\left(M_{5}\right)^{2}+\left(\frac{E}{2}\right)^{2} .
$$

Then

$$
\omega_{n}(\vec{k})=\left[n+\frac{1}{2}\right] E \pm\left[\left(m_{4}\right)^{2}+\vec{k}^{2}\right]^{1 / 2} .
$$

We see that apart from a momentum independent shift the spectrum is in this case exactly Lorentzian. The appearance of two branches is not alarming. These are just the usual particle antiparticle branches. It should be emphasized that it is quite possible to keep $m_{4}$ small (compared to $E$ ). This requires that the five dimensional mass term be tachyonic. (e.g., $\left(M_{5}\right)^{2}=-E^{2} / 4$ for massless matter). For some closing comments, note that there is no need for the five dimensional "Electromagnetism" considered here to have anything to do with ordinary electromagnetism. In addition, the total action of the field configuration considered here is zero.

$$
S=\int \mathrm{d}^{5} x \sqrt{-g}(R+2 \lambda+2 \mathcal{L})=\int \mathrm{d}^{5} x \sqrt{-g}\left(-2 E^{2}+E^{2}+E^{2}\right)=0 .
$$


Thus we expect field configurations of this type to be important for five dimensional quantum gravity even though they are not asymptotically flat.

Conclusion. We have looked at a general class of exotic Kaluza-Klein models in which the particles of the observable world are gravitationally trapped on a four dimensional submanifold of the "real" world. We have seen that it is generally possible to get a low energy spectrum that approximately respects Lorentz invariance, even though the metric ansatz does not possess an $O(1,3)$ symmetry. Finally we have exhibited a particular model in five dimensions that illustrates the discussed phenomena. The five dimensional model is interesting in other respects. It exhibits an interesting mechanism for cancelling a five dimensional cosmological constant. In addition it exhibits a mechanism for taking what would be a tachyon in flat five dimensional spacetime and producing an ordinary particle in the four dimensional submanifold.

This work was supported by the Division of High Energy Physics of the U.S. Department of Energy under contract DE-FG03-84ER40168.

References

[1] V.A. Rubakov and M.E. Shaposhnikov, Phys. Lett. 125B (1983) 136. [2] M.M. Nieto, Phys. Rev. A 17 (1978) 1273.

\section{Note added 14 years later:}

Submanifold variants of Kaluza-Klein theory, in which the $(3+1)$ dimensional matter is somehow constrained to live on a four-dimensional submanifold of a higher dimensional spacetime have a very long history, going back at least as far as Joseph [1]. Interest in this approach in a particle physics context, using non-gravitational fields to effect the trapping, is largely due to the early paper of Rubakov and Shaposhnikov [2].

After initial appearance of this present paper [3], the approach was somewhat modified by Squires [4]. Important related papers that further develop this type of approach are those of Laguna-Castillo and Matzner [5], and of Gibbons and Wiltshire [6]. The field then lay largely fallow for several years [7, 8, 9, 10, 11], until the appearance of the review article by Overduin and Wesson [12], which compares and contrasts some of the non-standard 
approaches to Kaluza-Klein gravity that have been considered in the literature.

In the last year and a half interest has picked up considerably [13, 114, 15, 16, 17, 18, 19, 20, 21]. Note that this topic is one for which e-prints are widely scattered, appearing on any one of the hep-th, hep-ph, or gr-qc archives.

Perhaps the best known of these recent papers are [18, 19, 21], which work specifically within a "brane" context arising from the low-energy limit of a fundamental string theory.? It should be borne in mind however, that many features of the "non-compact extra dimension" paradigm are generic to any low-energy effective field theory, and are largely independent of one's views regarding the full-fledged theory of quantum gravity.

\section{References}

[1] D.W. Joseph, "Coordinate covariance and the particle spectrum", Phys. Rev. 126, 319 (1962).

[2] V.A. Rubakov and M.E. Shaposhnikov, "Do we live inside a domain wall?", Phys. Lett. 125B (1983) 136.

[3] M. Visser, "An Exotic Class Of Kaluza-Klein Models", Phys. Lett. 159B, 22 (1985).

[4] E.J. Squires, "Dimensional Reduction Caused By A Cosmological Constant", Phys. Lett. 167B, 286 (1986).

[5] P. Laguna-Castillo and R.A. Matzner, "Surfaces of discontinuity in five dimensional Kaluza-Klein models", Nucl. Phys. B282, 542 (1987)

[6] G.W. Gibbons and D.L. Wiltshire, "Space-Time As A Membrane In Higher Dimensions", Nucl. Phys. B287, 717 (1987).

[7] T. Hubsch, "All The String's Vacua", Based on lectures given at the XIII International School of Theoretical Physics, Szczyrk, Poland, Sep 18-26, 1989.

[8] M.D. Maia and V. Silveira, "A Window to extra dimensions near a black hole", Phys. Rev. D48, 954 (1993).

\footnotetext{
${ }^{2}$ There are many other related papers and I am being highly selective here.
} 
Exotic Kaluza-Klein...

[9] U. Bleyer, M. Rainer and A. Zhuk, "A possible solution to the problem of extra dimensions", gr-qc/9503018.

[10] M.D. Maia, "Hypersurfaces of Five Dimensional Vacuum Space-times", gr-qc/9512002.

[11] A. Davidson and U. Paz, "Extensible Black Hole Embeddings for Apparently Forbidden Periodicities", gr-qc/9601023.

[12] J.M. Overduin and P.S. Wesson, "Kaluza-Klein gravity", Phys. Rept. 283, 303 (1997) gr-qc/9805018.

[13] M.D. Maia and E.M. Monte, "The dynamics of relativistic hypersurfaces", hep-th/9810028.

[14] G. Kalbermann and H. Halevi, "Nearness through an extra dimension", gr-qc/9810083.

[15] M. Gogberashvili, "Hierarchy problem in the shell-universe model", hep$\mathrm{ph} / 9812296$.

[16] M. Gogberashvili, "Our world as an expanding shell", hep-ph/9812365.

[17] M. Gogberashvili, "Four dimensionality in non-compact Kaluza-Klein model", hep-ph/9904383.

[18] L. Randall and R. Sundrum, "An alternative to compactification", hepth/9906064.

[19] C. Csaki, M. Graesser, C. Kolda and J. Terning, "Cosmology of one extra dimension with localized gravity", hep-ph/9906513.

[20] M. Gogberashvili, "Gravitational trapping for extended extra dimension", hep-ph/9908347.

[21] C. Csaki and Y. Shirman, "Brane junctions in the Randall-Sundrum scenario", hep-th/9908186. 